\title{
The integrated polarization of spiral galaxies
}

\author{
Jeroen M. Stil ${ }^{1}$, Marita Krause ${ }^{2}$, Lydia Mitchell ${ }^{1}$, Rainer Beck ${ }^{2}$ \\ and A. Russell Taylor ${ }^{1}$ \\ 1 Centre for Radio Astronomy, The University of Calgary, Calgary AB T2N 1N4, Canada \\ ${ }^{2}$ Max Planck Institut für Radioastronomie, Auf dem Hügel 69, Bonn, Germany
}

\begin{abstract}
We present deep observations of polarized radio emission of distant spiral galaxies with the Effelsberg 100-m telescope $\dagger$ at $4.8 \mathrm{GHz}$. These cross scans with sensitivity of $50 \mu \mathrm{Jy}$ or better open the possibility of a statistical study of magnetic field properties and internal Faraday rotation as a function of inclination for a large sample of unresolved galaxies. The Square Kilometre Array (SKA) will be able to detect polarization of spiral galaxies to high redshift, probing the evolution of magnetic fields in disk galaxies over cosmic time.
\end{abstract}

Keywords. Galaxies: magnetic fields - galaxies: spiral - radio continuum: galaxies - polarization

\section{Integrated polarization of spiral galaxies}

The regular azimuthal magnetic field in spiral galaxy disks leads to polarized synchrotron emission, with locally observed values up to $40 \%$ polarized at $4.8 \mathrm{GHz}$. When an unresolved galaxy is observed at an angle with respect to the plane of the disk, the regular magnetic field projects into a net component in the plane of the sky, resulting in net polarized emission for the galaxy as a whole. Stil et al. (2008) presented observations and models of the polarization of the integrated radio emission of spiral galaxies at 4.8 $\mathrm{GHz}$, with fractional polarization up to $17 \%$. Figure 1 shows the sample of spiral galaxies without a bar (a) and with a bar (b) from Stil et al. (2008).

The curves in Figure 1 (a) and (b) represent axially symmetric models for the integrated polarization of spiral galaxies by Stil et al. (2008) that include depolarization by wavelength-independent depolarization, Faraday dispersion, and differential Faraday rotation (Sokoloff et al. 1998). Parameters in the model are the regular magnetic field $B$, the ratio of random to regular magnetic field strength $f_{\mathrm{B}}$, electron density $n_{e}$ with filling factor $f_{i}$, thickness of the disk $2 h$, and a turbulent scale length for rotation measure fluctuations $l_{\text {turb }}$. A mean thermal fraction of $23 \%$ is assumed at $4.8 \mathrm{GHz}$ (Condon $\&$ Yin 1990). From inclination $0^{\circ}$ (face-on), to higher inclination, the degree of polarization initially increases as the regular magnetic field projects into a net component of the magnetic field in the plane of the sky. At inclinations higher than $\sim 60^{\circ}$, Faraday depolarization dominates and the degree of polarization decreases with inclination.

The axially symmetric models successfully reproduce the observations in Figure 1a, but not in Figure 1b where large-scale magnetic fields along the bar add the orientation of the bar as an additional parameter that is currently not included in the models. An interesting feature of the models is the convergence of curves with the same value of $f_{\mathrm{B}}$ for inclinations less than $50^{\circ}$. The uniformity of the magnetic field defines the polarized intensity almost everywhere in the disk, and thus the degree of polarization of

$\dagger$ The Effelsberg 100-m telescope is operated by the MPIfR on behalf of the Max-Planck-Gesellschaft 

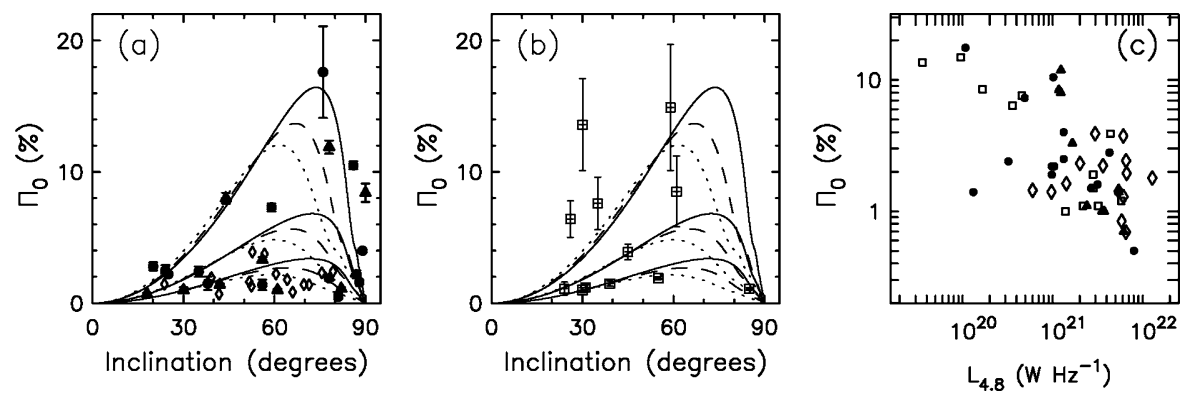

Figure 1. (a) Integrated fractional polarization at $4.8 \mathrm{GHz}$ of nearby spiral galaxies (dots), Virgo cluster spirals (triangles), and new Effelsberg observations of distant UGC spiral galaxies (diamonds). The curves represent axially symmetric models of the integrated polarization that include wavelength-independent depolarization Faraday dispersion and differential Faraday rotation. Upper solid curve: $B=5 \mu \mathrm{G}, f_{\mathrm{B}}=1.0, n_{e}=0.03 \mathrm{~cm}^{-3}, f_{i}=0.5, l_{\text {turb }}=50 \mathrm{pc}$, $(2 h)=1000 \mathrm{pc}$, thermal fraction is 0.23 . Dashed curve: double the magnetic field $B$. Dotted curve: triple the electron density $n_{e}$. (b) The same as (a) for barred galaxies (squares). The model curves in (b) are shown for comparison with (a) only. (c) Anti-correlation between fractional polarization and $4.8 \mathrm{GHz}$ luminosity.

the integrated emission. This is a feature of models at frequencies more than a few GHz, where Faraday dispersion and differential Faraday rotation are relatively unimportant for low inclinations.

Figure 1c shows an anti-correlation of fractional polarization of the integrated emission with radio luminosity. This anti-correlation suggest that the magnetic field of luminous galaxies is less uniform, possibly because of a higher star formation rate.

Figure 1 also shows 14 galaxies observed in deep cross scans at $4.8 \mathrm{GHz}$ with the Effelsberg telescope shown as diamonds. These galaxies are Milky Way size spiral galaxies at distances of several tens of Mpc selected from the Uppsala General Catalog (UGC) and detected in the NVSS with a $1.4 \mathrm{GHz}$ flux density of $20 \mathrm{mJy}$ or higher. The angular size of these galaxies is smaller than the $2.5^{\prime}$ beam of the Effelsberg telescope at 4.8 $\mathrm{GHz}$. A sensitivity of $50 \mu \mathrm{Jy}_{\text {beam }}{ }^{-1}$ can be obtained with 1 hour total observing time per galaxy. This makes it possible to observe a large sample of galaxies for a statistical analysis of magnetic field uniformity and Faraday depolarization.

\section{Evolution of Magnetic fields in disk galaxies}

The Square Kilometre Array (SKA) will be able to detect polarization of radio sources fainter than $100 \mu \mathrm{Jy}$, most of which are distant star forming galaxies. Although highredshift galaxies will be mostly unresolved, polarization of their integrated emission provides information on the uniformity of their magnetic field and the amount of Faraday rotation. Our Effelsberg observations, when completed, will be part of a local comparison sample for galaxies at high redshift. Such observations will test large-scale dynamo models such as the turbulent dynamo at high redshift (Arshakian et al. 2008).

\section{References}

Arshakian, T., Beck, R., Krause, M., \& Sokoloff, D., 2008, A\&A, in press, arXiv 0810.3114 Condon, J. J. \& Yin, Q. F. 1990, ApJ 357, 97

Condon, J. J., Cotton, W. D., Greisen, E. W., et al. 1998, AJ 115, 1693

Sokoloff, D. D., Bykov, A. A., Shukurov, A., et al. 1998, MNRAS 299, 189

Stil, J. M., Krause, M., Beck, R., \& Taylor, A. R. 2008, ApJ, in press arXiv 0810.2303 